\title{
An extended power Lindley distribution and its application
}

\author{
Ibrahim Elbatal ${ }^{1}$, Yehia Mousa Hussein El Gebaly ${ }^{2}$ and Essam Ali Amin Abd \\ El- Samad ${ }^{1,3, *}$ \\ ${ }^{1}$ Department of Mathematics and Statistics, College of Science Al Imam Mohammad Ibn Saud Is- \\ lamic University, Saudi Arabia \\ ${ }^{2}$ Department of Statistics, Mathematics and Insurance, Benha University \\ ${ }^{3}$ Institute of Statistical Studies and Research, Department of Mathematical Statistics, Cairo Uni- \\ versity, Egypt
}

Received October 16, 2016; December 27, 2016

Copyright (c) 2016, Afrika Statistika and Statistics and Probability African Society (SPAS). All rights reserved

\begin{abstract}
This paper introduces a new five-parameter lifetime model called the exponentiated Kumaraswamy power Lindley distribution, that extends the power Lindley distribution and some well-known distributions. Various structural properties of the new model including expansions for the density function, explicit expressions for the ordinary and conditional moments, residual and reversed residual life functions and mean deviations are derived. The maximum likelihood method is used to estimate the model parameters. The usefulness and flexibility of the proposed model are illustrated empirically by means of two real data sets.

Résumé. Ce papier introduit un modèle de durée de vie à cinq variables appelée exponentiation du modèle puissance Kumaraswamy de Lindley, qui généralise le modèle puissance de la distribution de Lindley et cetaines distribitions connues. Y sont établies différentes propriétés structurelles di mouveau modèle, y compris des dévelopments en série de la densité de probabilité, une expression explicite des moments conditionnels et inconditionnels, les fonction de durée de vie (résiduelles et résiduelles inverses), et des propriétés relatives à la déviation par rapport à la moyenne. Les paramètres ont été estimés par la méthode du maximum de vraisemblance. L'utilité et la flexibility de la méthode a été montrée sur deux jeux de données réelles.
\end{abstract}

Key words: Exponentiated Kumaraswamy-G; Maximum likelihood estimation; Moments; Power Lindley distribution.

AMS 2010 Mathematics Subject Classification : 60E05; 62 F12.

*Corresponding author Essam Ali Amin Abd El- Samad : eaabdelsamad@imamu.edu.sa Yehia Mousa Hussein El Gebaly : yehia1958@hotmail.com

Ibrahim Elbatal : iielbatal@imamu.edu.sa 


\section{Introduction}

In many applied sciences such as medicine, engineering and finance, amongst others, modeling and analyzing lifetime data are crucial. Several lifetime distributions have been used to model such kinds of data. For instance, the exponential, Weibull, gamma, Rayleigh distributions and their generalizations (Gupta and Kundu, 1999). Each distribution has its own characteristics due specifically to the shape of the failure rate function which may be only monotonically decreasing or increasing or constant in its behavior, as well as non-monotone, being bathtub shaped or even unimodal.

The Lindley ( $\mathrm{Li}$ ) distribution was introduced by Lindley (1958) as a new distribution useful to analyse lifetime data especially in applications modeling stress-strength reliability. In a recent paper Ghitany et al. (2008) studied the properties of the Li distribution under a carefully mathematical treatment. They also showed in a numerical example that the Li distribution gives better modeling for waiting times and survival times data than the exponential distribution. The use of the $\mathrm{Li}$ distribution could be a good alternative to analyse lifetime data within the competing risks approach as compared with the use of standard exponential or even the Weibull distribution commonly used in this area. The exponential distribution assumes constant hazard function, usually not an appropriated assumption for many competing risks data. The Li distribution has some nice properties to be used in lifetime data analysis as closed forms for the survival and hazard functions and good flexibility of fit.

The probability density function (pdf) and cumulative distribution function (cdf) of the Li model are given (for $x>0$ ) by

$$
g(x)=\frac{\alpha^{2}}{\alpha+1}(1+x) e^{-\alpha x} \text { and } G(x)=1-e^{-\alpha x}\left(1+\frac{\alpha x}{\alpha+1}\right),
$$

where $\alpha>0$ is a scale parameter.

In the context of reliability studies, Ghitany et al. (2013) proposed a more flexible distribution for modeling lifetime data called power $\mathrm{Li}(\mathrm{PLi})$ distribution which is extends the $\mathrm{Li}$ distribution. It can accommodate both decreasing and increasing failure rates as its antecessors, as well as unimodal and bathtub shaped failure rates. The pdf and cdf of the PLi distribution are given (for $x>0$ ) by

$$
g(x)=\frac{\alpha^{2} \theta}{1+\alpha}\left(1+x^{\theta}\right) x^{\theta-1} e^{-\alpha x^{\theta}} \text { and } G(x)=1-\left(1+\frac{\alpha x^{\theta}}{\alpha+1}\right) e^{-\alpha x^{\theta}},
$$

where $\alpha>0$ is a scale parameter and $\theta>0$ is a shape parameter.

The distribution by Kumaraswamy (Kw) (Kumaraswamy, 1980) is not very common among statisticians and has been little explored in the literature. Its pdf and cdf are given (for

Journal home page: www.jafristat.net ; www.projecteuclid.org/as 
$0<x<1)$ by

$$
f(x, a, b)=a b x^{a-1}\left(1-x^{a}\right)^{b-1} \text { and } F(x)=1-\left(1-x^{a}\right)^{b},
$$

where $a$ and $b$ are positive shape parameters. This model can be unimodal, increasing, decreasing or constant, depending on the parameter values. It does not seem to be very familiar to statisticians and has not been investigated systematically in much detail before, nor has its relative interchangeability with the beta distribution been appreciated. Recently, Cordeiro and de Castro (2011) explored the background and genesis of this distribution and, more importantly, made clear some similarities and differences between the beta and Kw distributions.

For an arbitrary parent cdf $G(x)$, Ghitany et al. (2008) defined the Kw-G class of distributions by the cdf $F(x)$ and pdf $f(x)$

$$
F(x)=1-\left[1-G(x)^{a}\right]^{b} \text { and } f(x)=\operatorname{abg}(x) G(x)^{a-1}\left[1-G(x)^{a}\right]^{b-1},
$$

where $a$ and $b$ are two additional positive shape parameters. The Kw-G distribution can be used quite effectively even if the data are censored. Correspondingly, its density function is distributions has a very simple form. The pdf (2) has many of the same properties of the class of beta-G family (Eugene et al., 2002), but has some advantages in terms of tractability, since it does not involve any special function such as the beta function. Equivalently, as occurs with the beta-G family of distributions, special $\mathrm{Kw}-\mathrm{G}$ distributions can be generated as follows: the Kw-normal distribution is obtained by taking $G(x)$ in $(2)$ to be the normal cumulative function. Analogously, the $\mathrm{Kw}$-Weibull (Cordeiro et al., 2010), Kw-generalized gamma (Pascoa et al., 2011), Kw-Birnbaum-Saunders (Saulo et al., 2012), Kw-Gumbel (Cordeiro et al., 2012) and Kw-complementary Weibull geometric (Afify et al., 2016a) distributions are obtained by taking $G(x)$ to be the cdf of the Weibull, generalized gamma, Birnbaum-Saunders, Gumbel and complementary Weibull geometric distributions, respectively, among several others.

Since 1995, the exponentiated distributions have been widely studied in statistics and numerous authors have developed various classes of these distributions Mudholkar et al. (1995) proposed the exponentiated Weibull distribution. Gupta and Kundu (1999) introduced the exponentiated exponential distribution as a generalization of the standard exponential distribution. Nadarajah and Kotz (2006) proposed, based on the same idea, four more exponentiated type distributions to extend the standard gamma, standard Weibull, standard Gumbel and standard Fréchet distributions.

Recently, Lemonte et al. (2013) proposed a generalization of the Kumaraswamy distribution, so-called the exponentiated Kumaraswamy-G (EK-G) family. The cdf and pdf of the EK-G class are given by

$$
F(x)=\left\{1-\left[1-G(x)^{a}\right]^{b}\right\}^{c}
$$

and

$$
f(x)=\operatorname{abcg}(x) G(x)^{a-1}\left[1-G(x)^{a}\right]^{b-1}\left\{1-\left[1-G(x)^{a}\right]^{b}\right\}^{c-1}
$$

Journal home page: www.jafristat.net ; www.projecteuclid.org/as 
where $a, b$ and $c$ are positive shape parameters. Huang and Oluyede (2014) have used the cdf of Dagum distribution to propose the exponentiated Kumaraswamy-Dagum distribution. Rodrigues et al. (2015) introduced the exponentiated Kumaraswamy exponential distribution. Rodrigues et al. (2016) presented the exponentiated Kumaraswamy inverse Weibull distribution with application in survival analysis.

Many authors defined generalizations of the Li distribution. For example, the two-parameter weighted Li [Ghitany et al. (2011)], generalized Li [Nadarajah et al. (2011)], extended Li (Bakouch et al., 2012), beta Li (BLi) (Merovci and Sharma, 2014), Kumaraswamy Li (KLi) (Cakmakyapan and Kadilar, 2014), transmuted Li-geometric (Merovci and Elbatal , 2014), beta-exponentiated power Li (Pararai et al., 2015), gamma Li (Nedjar and Zeghdoudi, 2016), beta transmuted Li (Afify et al., 2016b), odd log-logistic Li (Ozel et al., 2016), complementary generalized transmuted Poisson Li (CGTPLi) (Alizadeh et al., 2016) and pseudo Li (Zeghdoudi and Nedjar, 2016) distributions.

The aim of this paper is to define and study a new lifetime model called the exponentiated Kumaraswamy power Lindley (EKPLi) distribution. Its main feature is that three additional shape parameters are inserted in equation (1) to provide more flexibility for the generated model. Using the EK-G family of distributions (Lemonte et al., 2013), we construct the five-parameter EKPLi model and give a comprehensive description of some of its mathematical properties.

The rest of the article is organized as follows. In Section 2, we define the EKPLi distribution and provide some plots for its pdf, cdf and hazard rate function (hrf). We also derive a useful linear representation for its pdf in this section. We obtain, in Section 3, some mathematical properties of the new distribution including ordinary and conditional moments, moments of the residual life and reversed residual life, Bonferroni and Lorenz curves and mean deviations. The maximum likelihood estimates (MLEs) of the unknown model parameters are provided in Section 4. In Section 5, we prove empirically the EKPLi flexibility by means of two real data sets. Finally, in Section 6,we provide some concluding remarks.

\section{The EKPLi model}

In this section, we define the five-parameter EKPLi distribution. By inserting equation (1) in equation (3), we obtain the cdf of the EKPLi distribution

$$
F(x)=\left(1-\left\{1-\left[1-\left(1+\frac{\alpha x^{\theta}}{\alpha+1}\right) e^{-\alpha x^{\theta}}\right]^{a}\right\}^{b}\right)^{c}
$$

The corresponding pdf is given by

$$
\begin{aligned}
f(x)= & \frac{a b c \alpha^{2} \theta}{1+\alpha}\left[1-\left(1+\frac{\alpha x^{\theta}}{\alpha+1}\right) e^{-\alpha x^{\theta}}\right]^{a-1}\left\{1-\left[1-\left(1+\frac{\alpha x^{\theta}}{\alpha+1}\right) e^{-\alpha x^{\theta}}\right]^{a}\right\}^{b-1} \\
& \times\left(1+x^{\theta}\right) x^{\theta-1} e^{-\alpha x^{\theta}}\left(1-\left\{1-\left[1-\left(1+\frac{\alpha x^{\theta}}{\alpha+1}\right) e^{-\alpha x^{\theta}}\right]^{a}\right\}^{b}\right)^{c-1} \cdot
\end{aligned}
$$


Henceforth, let $X \sim \operatorname{EKPLi}(\alpha, \theta, a, b, c)$ be a random variable with density function (6). In fact, the EKPLi model can provide better fits than at least ten other nested and non-nested models, in two applications. The EKPLi distribution, as shown in the application section, is cabable formodelling left skewd data.

The EKPLi distribution has an attractive physical interpretation whenever $a, b$ and $c$ are positive integers. Suppose a device is made up of $c$ independent components in a series system. Furthermore, each component is made of $a$ independent subcomponents identically distributed according to $\left[1-\left(1+\frac{\alpha x^{\theta}}{\alpha+1}\right) e^{-\alpha x^{\theta}}\right]^{a}$ in a parallel system. So, the device fails if any of the $b$ components fail and each component fails if all of its $a$ subcomponents fail. If the sub-component lifetimes have a common $\left[1-\left(1+\frac{\alpha x^{\theta}}{\alpha+1}\right) e^{-\alpha x^{\theta}}\right]^{a}$ cumulative function, then the lifetime of the device will follow the EKPLi distribution (5).

The reliability function (sf) and hazard rate function (hrf) of $X$ are given by

$$
R(x)=1-\left(1-\left\{1-\left[1-\left(1+\frac{\alpha x^{\theta}}{\alpha+1}\right) e^{-\alpha x^{\theta}}\right]^{a}\right\}^{b}\right)^{c}
$$

and

$$
\begin{aligned}
h(x)= & \frac{a b c \alpha^{2} \theta}{1+\alpha}\left[1-\left(1+\frac{\alpha x^{\theta}}{\alpha+1}\right) e^{-\alpha x^{\theta}}\right]^{a-1}\left\{1-\left[1-\left(1+\frac{\alpha x^{\theta}}{\alpha+1}\right) e^{-\alpha x^{\theta}}\right]^{a}\right\}^{b-1} \\
& \times\left(1+x^{\theta}\right) x^{\theta-1} e^{-\alpha x^{\theta}} \frac{\left(1-\left\{1-\left[1-\left(1+\frac{\alpha x^{\theta}}{\alpha+1}\right) e^{-\alpha x^{\theta}}\right]^{a}\right\}^{b}\right)^{c-1}}{1-\left(1-\left\{1-\left[1-\left(1+\frac{\alpha x^{\theta}}{\alpha+1}\right) e^{-\alpha x^{\theta}}\right]^{a}\right\}^{b}\right)^{c}}
\end{aligned}
$$

The EKPLi distribution is very flexible model that approaches to different distributions when its parameters are changed. The EKPLi distribution contains 7 sub-models listed in Table 1. Figure 1 displays some plots of the EKPLi density for some values of the parameters $\theta, a, b$ and $c$ with $\alpha=1$. Further, the hrf of the new distribution is very flexible (see Figure 2 ) and thus it becomes an important model to fit real lifetime data. The plots of the EKPLi cdf are displayed in Figure 2.

\subsection{Linear Representation}

In this section, we provide some representation of the pdf of EKPLi distribution. The mathematical relation given below will be useful in this subsection.

By using the generalized binomial expansion if $\beta$ is a positive and $|z|<1$, then

$$
(1-z)^{\beta-1}=\sum_{i=0}^{\infty}(-1)^{i}\left(\begin{array}{c}
\beta-1 \\
i
\end{array}\right) z^{i}
$$


I. Elbatal, Y.M.H. El Gebaly and E.A. Amin, Afrika Statistika, Vol. 11(2), 2016, pages 1075 1094. An extended power Lindley distribution and its application.

\begin{tabular}{cccccccc}
\hline \hline \multicolumn{1}{c}{ Reduced } & \multicolumn{7}{c}{ Parameters } \\
No. & model & $\alpha$ & $\theta$ & $a$ & $b$ & $c$ & Author(s) \\
\hline 1 & $\mathrm{EKLi}$ & $\alpha$ & 1 & $a$ & $b$ & $c$ & New \\
2 & $\mathrm{KPLi}$ & $\alpha$ & $\theta$ & $a$ & $b$ & 1 & - \\
3 & $\mathrm{EPLi}$ & $\alpha$ & $\theta$ & $a$ & 1 & 1 & Liyanage and Pararai (2014) \\
4 & $\mathrm{PLi}$ & $\alpha$ & $\theta$ & 1 & 1 & 1 & Ghitany et al. $(2013)$ \\
5 & $\mathrm{GLi}$ & $\alpha$ & 1 & $a$ & 1 & 1 & Nadarajah et al. $(2011)$ \\
6 & $\mathrm{KLi}$ & $\alpha$ & 1 & $a$ & $b$ & 1 & Cakmakyapan and Kadilar (2014) \\
7 & $\mathrm{Li}$ & $\alpha$ & 1 & 1 & 1 & 1 & Lindley (1958) \\
\hline \hline
\end{tabular}

Table 1. Special models of the EKPLi distribution
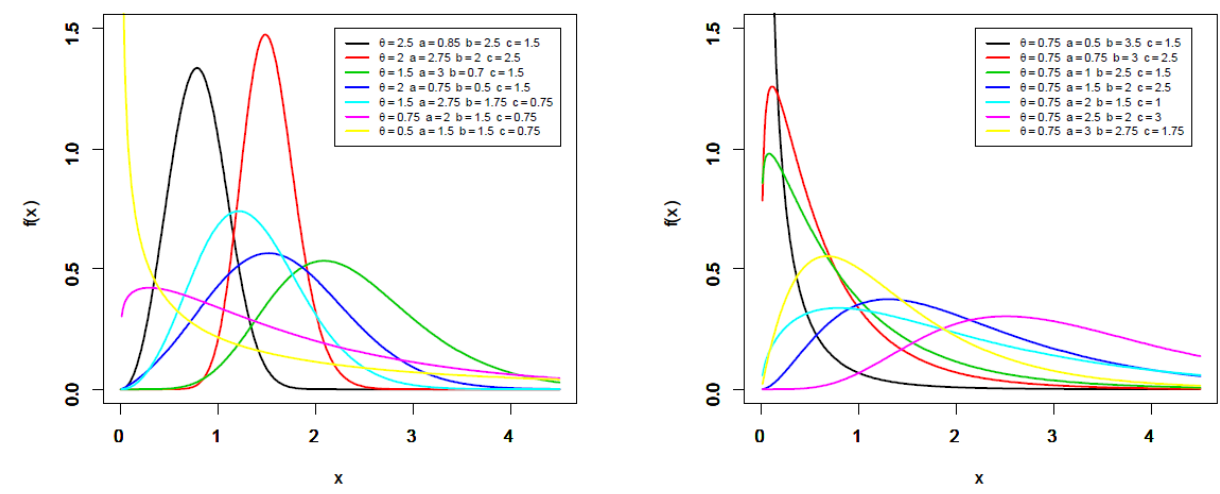

Fig. 1. Plots of the EKPLi pdf for some parameters values
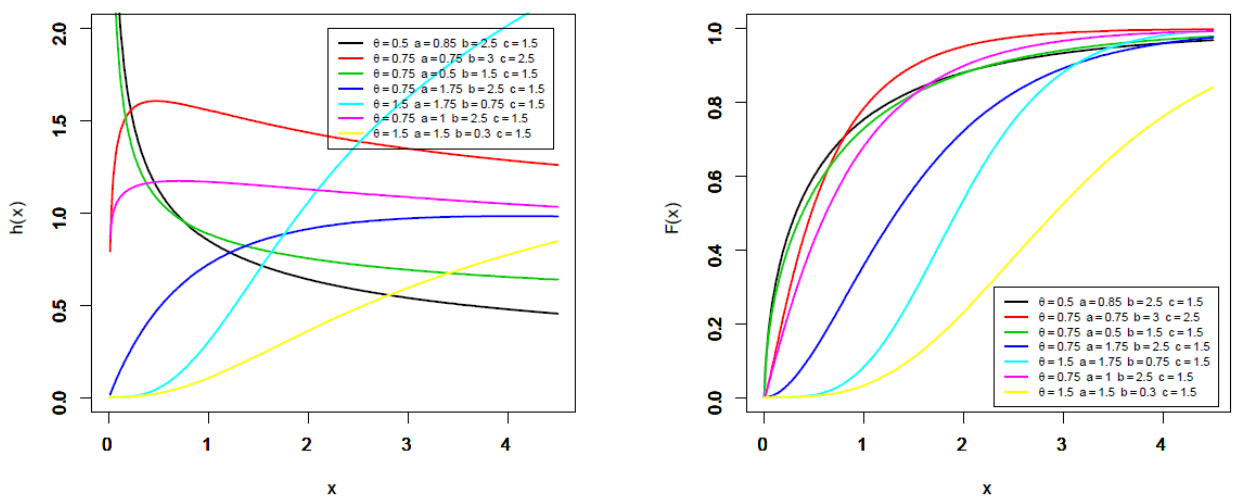

Fig. 2. Plots of the EKPLi hrf (left panel) and cdf (right panel) for some

Using (7) the equation (6) becomes 


$$
\begin{aligned}
f(x)= & \frac{a b c \alpha^{2} \theta}{1+\alpha} \sum_{i, j, k=0}^{\infty}(-1)^{i+j+k}\left(\begin{array}{c}
c-1 \\
i
\end{array}\right)\left(\begin{array}{c}
b(i+1)-1 \\
j
\end{array}\right)\left(\begin{array}{c}
a(j+1-1 \\
k
\end{array}\right) \\
& \times\left(1+x^{\theta}\right) x^{\theta-1}\left(1+\frac{\alpha x^{\theta}}{\alpha+1}\right)^{k} e^{-\alpha(k+1) x^{\theta}}
\end{aligned}
$$

Using the binomial expansion for the term $\left(1+\frac{\alpha x^{\theta}}{\alpha+1}\right)^{k}$, we obtain

$$
\left(1+\frac{\alpha x^{\theta}}{\alpha+1}\right)^{k}=\sum_{j=0}^{l}\left(\frac{\alpha}{\alpha+1}\right)^{l} x^{\theta l}
$$

Thus, equation (8) reduces to

$$
f(x)=\sum_{k=0}^{\infty} \sum_{j=0}^{l} \eta_{k, l}\left(x^{\theta(l+1)-1}+x^{\theta(l+2)-1}\right) e^{-\alpha(k+1) x^{\theta}},
$$

where

$$
\eta_{k, l}=\frac{a b c \alpha^{2} \theta}{1+\alpha} \sum_{i, j=0}^{\infty}(-1)^{i+j+k}\left(\begin{array}{c}
c-1 \\
i
\end{array}\right)\left(\begin{array}{c}
b(i+1)-1 \\
j
\end{array}\right)\left(\begin{array}{c}
a(j+1-1 \\
k
\end{array}\right)\left(\frac{\alpha}{\alpha+1}\right)^{l}
$$

\section{Mathematical properties}

In this section we studied some mathematical properties of the EKPLi distribution including moments, conditional moments, residual and reversed residual life functions, Bonferroni and Lorenz curves and mean deviation.

Lemma 1: If $X$ has $\operatorname{EKPLi}(\varphi)$ where $\varphi=(\alpha, \theta, a, b, c)$, then the $r$ th moment of $X$ is given by

$$
\mu_{r}^{\prime}=\sum_{k=0}^{\infty} \sum_{j=0}^{l} \eta_{k, l} \frac{\Gamma\left(\frac{r}{\theta}+l+1\right)}{\theta(\alpha(k+1))^{\frac{r}{\theta}+l+1}}\left[1+\frac{\frac{r}{\theta}+l+1}{(\alpha(k+1))}\right] .
$$

\section{Proof.}

The $r$ th ordinary moment of the EKPLi distribution is given by

$$
\begin{aligned}
\mu_{r}^{\prime} & =\int_{0}^{\infty} x^{r} f(x, \varphi) d x \\
& =\sum_{k=0}^{\infty} \sum_{j=0}^{l} \eta_{k, l} \int_{0}^{\infty}\left(x^{r+\theta(l+1)-1}+x^{r+\theta(l+2)-1}\right) e^{-\alpha(k+1) x^{\theta}} d x \\
& =\sum_{k=0}^{\infty} \sum_{j=0}^{l} \eta_{k, l} \frac{\Gamma\left(\frac{r+\theta(l+1)}{\theta}\right)}{\theta(\alpha(k+1))^{\frac{r+\theta(l+1)}{\theta}}}\left[1+\frac{\frac{r+\theta(l+1)}{\theta}}{(\alpha(k+1))}\right],
\end{aligned}
$$

Journal home page: www.jafristat.net; www.projecteuclid.org/as 
which completes the proof .

The central moments $\mu_{r}$ and cumulants $\kappa_{r}$ of the EKPLi distribution can be determined from expression (10) as $\mu_{r}=\sum_{m=0}^{r}\left(\begin{array}{c}r \\ m\end{array}\right)(-1)^{m} \mu_{1}^{\prime m} \mu_{r-m}^{\prime}$ and $\kappa_{r}=\mu_{r}^{\prime}-\sum_{m=1}^{r-1}\left(\begin{array}{c}r-1 \\ m-1\end{array}\right) \kappa_{m}$ $\mu_{r-m}^{\prime}$, respectively, where $\kappa_{1}=\mu_{1}^{\prime}, \kappa_{2}=\mu_{2}^{\prime}-\mu_{1}^{/ 2}, \kappa_{3}=\mu_{3}^{\prime}-3 \mu_{2}^{\prime} \mu_{1}^{\prime}+2 \mu_{1}^{/ 3}$, and $\kappa_{4}=\mu_{4}^{\prime}-4 \mu_{1}^{\prime} \mu_{3}^{\prime}-3 \mu_{2}^{/ 2}+12 \mu_{2}^{\prime} \mu_{1}^{/ 2}-6 \mu_{1}^{/ 4}$,etc. Additionally, the skewness and kurtosis can be calculated from the third and fourth standardized cumulants in the forms $S K=\frac{\kappa_{3}}{\sqrt{\kappa_{2}^{3}}}$ and $K U=\frac{\kappa_{4}}{\kappa_{2}^{2}}$, respectively.

The moment generating function $M_{X}(t)$ is given by

$$
M_{X}(t)=\sum_{k, r=0}^{\infty} \sum_{j=0}^{l} \eta_{k, l} \frac{t^{r}}{r !} \frac{\Gamma\left(\frac{r+\theta(l+1)}{\theta}\right)}{\theta(\alpha(k+1))^{\frac{r+\theta(l+1)}{\theta}}}\left[1+\frac{\frac{r+\theta(l+1)}{\theta}}{(\alpha(k+1))}\right] .
$$

\subsection{Conditional Moments}

For lifetime models, it is also of interest to find the conditional moments and the mean residual lifetime function. The conditional moments for EKPLi distribution is given by the following Lemma.

Lemma 1. If $X$ has EKPLi $(\varphi)$, the conditional moments for EKPLi distribution is given by

$$
\nu_{s}=E\left(X^{s} \mid X>t\right)=\sum_{k=0}^{\infty} \sum_{j=0}^{l} \eta_{k, l}\left[\frac{\Gamma\left(\frac{s}{\theta}+l+1, \alpha(k+1) t^{\theta}\right)}{\theta(\alpha(k+1))^{\frac{s}{\theta}+l+1}}+\frac{\Gamma\left(\frac{s}{\theta}+l+2, \alpha(k+1) t^{\theta}\right)}{\theta(\alpha(k+1))^{\frac{s}{\theta}+l+1}}\right] .
$$

\section{Proof.}

$$
\begin{aligned}
\nu_{s} & =E\left(X^{s} \mid X>t\right)=\int_{t}^{\infty} x^{s} f(x, \phi) d x \\
& =\sum_{k=0}^{\infty} \sum_{j=0}^{l} \eta_{k, l} \int_{t}^{\infty}\left(x^{s+\theta(l+1)-1}+x^{s+\theta(l+2)-1}\right) e^{-\alpha(k+1) x^{\theta}} d x \\
& =\sum_{k=0}^{\infty} \sum_{j=0}^{l} \eta_{k, l}\left[\frac{\Gamma\left(\frac{s}{\theta}+l+1, \alpha(k+1) t^{\theta}\right)}{\theta(\alpha(k+1))^{\frac{s}{\theta}+l+1}}+\frac{\Gamma\left(\frac{s}{\theta}+l+2, \alpha(k+1) t^{\theta}\right)}{\theta(\alpha(k+1))^{\frac{s}{\theta}+l+1}}\right],
\end{aligned}
$$

where $\Gamma(s, t)=\int_{t}^{\infty} x^{s-1} e^{-x} d x$ is the upper incomplete gamma function.

If we ask about how much longer will an component of age $t$ live? This question is vital for reliability analysis, survival analysis, actuarial applications and other disciplines. The mean residual lifetime function defines the mean lifetime left for an item of age $t$. Along with the failure rate, it plays a crucial role in reliability analysis, survival analysis, demography and other disciplines. In demography, for example, this important population characteristic is 
called the "life expectancy at time $t$ " and in risk analysis the term "mean excess time" is often used.

The mean residual lifetime function of $X$ is given by

$$
\begin{aligned}
\mu(t)= & E(X \mid X>t)-t=\frac{1}{\bar{F}(t)} \sum_{k=0}^{\infty} \sum_{j=0}^{l} \eta_{k, l} \\
& \times\left[\frac{\Gamma\left(\frac{1}{\theta}+l+1, \alpha(k+1) t^{\theta}\right)}{\theta(\alpha(k+1))^{\frac{1}{\theta}+l+1}}+\frac{\Gamma\left(\frac{1}{\theta}+l+2, \alpha(k+1) t^{\theta}\right)}{\theta(\alpha(k+1))^{\frac{1}{\theta}+l+1}}\right]-t .
\end{aligned}
$$

Also, the importance of the $M R L$ function is due to its uniquely determination of the lifetime distribution as well as the failure rate (FR) function. Lifetimes can exhibit $I M R L$ (increasing $M R L$ ) or $D M R L$ (decreasing $M R L)$ ). $M R L$ functions that first decreases (increases) and then increases (decreases) are usually called bathtub (upside-down bathtub) shaped, $B M R L$ $(U M R L)$. Several authors have been studied the relationship between the behaviors of the $M R L$ and $F R$ functions of a distribution. (See, Ghitany, 1998, Mi, 1995, Park, 1985 and Tang et al., 1999).

\subsection{Residual and reversed residual life functions}

In reliability theory and its applications deal with distributions of continuous and discrete lifetimes. The two commonly used measures of describing the lifetime of items are the mean residual life (MRL) or life expectancy and the failure rate functions. Lifetime distributions having decreasing, increasing, bathtub-shaped, or upside-down bathtub-shaped MRL are used to model various lifetime data which occur in many areas of science such as reliability, survival analysis, economics, actuarial, and many others. For example, in biomedical sciences, researchers analyze survivorship studies by using the MRL. The failure rate function also has numerous applications, including modelling the lifetime of electronic, electro-mechanical, and mechanical products. On the other hand, upside-down bathtubshaped failure rate functions, typified by failure due to fatigue, are commonly used for modelling lifetimes of mechanical parts and semiconductors.

Given that a component survives up to time $t \geq 0$, the residual life is the period beyond $t$ until the time of failure and defined by the conditional random variable $X-t \mid X>t$. In reliability, it is well known that the mean residual life function and ratio of two consecutive moments of residual life determine the distribution uniquely (Gupta and Gupta, 1983). Therefore, the $r$ th-order moment of the residual lifetime can be obtained via the general formula

$$
\mu_{r}(t)=E\left((X-t)^{r} \mid X>t\right)=\frac{1}{\bar{F}(t)} \int_{t}^{\infty}(x-t)^{r} f(x, \varphi) d x, r \geq 1
$$

Applying the binomial expansion of $(x-t)^{r}$ into the above formula, we get

Journal home page: www.jafristat.net ; www.projecteuclid.org/as 


$$
\begin{aligned}
\mu_{r}(t)= & \frac{1}{\bar{F}(t)} \sum_{k=0}^{\infty} \sum_{j=0}^{l} \sum_{d=0}^{r} \eta_{k, l}(-t)^{d}\left(\begin{array}{c}
r \\
d
\end{array}\right) \int_{t}^{\infty}\left(x^{r-d+\theta(l+1)-1}+x^{r-d+\theta(l+2)-1}\right) e^{-\alpha(k+1) x^{\theta}} d x \\
= & \frac{1}{\bar{F}(t)} \sum_{k=0}^{\infty} \sum_{j=0}^{l} \sum_{d=0}^{r} \eta_{k, l}(-t)^{d}\left(\begin{array}{c}
r \\
d
\end{array}\right) \\
& \times\left[\frac{\Gamma\left(\frac{r-d}{\theta}+l+1, \alpha(k+1) t^{\theta}\right.}{\theta(\alpha(k+1))^{\frac{r-d}{\theta}+l+1}}+\frac{\Gamma\left(\frac{r-d}{\theta}+l+2, \alpha(k+1) t^{\theta}\right)}{\theta(\alpha(k+1))^{\frac{r-d}{\theta}+l+2}}\right] .
\end{aligned}
$$

On the other hand, we analogously discuss the reversed residual life and some of its properties. The reversed residual life can be defined as the conditional random variable $t-X \mid X \leq t$ which denotes the time elapsed from the failure of a component given that its life is less than or equal to $t$. This random variable may also be called the inactivity time (or time since failure). Mreover, in reliability, the mean reversed residual life and ratio of two consecutive moments of reversed residual life characterize the distribution uniquely.

The $r$ th-order moment of the reversed residual life can be obtained by the well known formula

$$
m_{r}(t)=E\left((t-X)^{r} \mid X \leq t\right)=\frac{1}{F(t)} \int_{0}^{\infty}(t-x)^{r} f(x, \varphi) d x, r \geq 1 .
$$

Applying the binomial expansion of $(t-x)^{r}$ into the above formula gives

$$
\begin{aligned}
m_{r}(t)= & \frac{1}{F(t)} \sum_{k=0}^{\infty} \sum_{j=0}^{l} \sum_{d=0}^{r} \eta_{k, l}(-t)^{d}\left(\begin{array}{c}
r \\
d
\end{array}\right) \int t^{\infty}\left(x^{r-d+\theta(l+1)-1}+x^{r-d+\theta(l+2)-1}\right) e^{-\alpha(k+1) x^{\theta}} d x \\
= & \frac{1}{F(t)} \sum_{k=0}^{\infty} \sum_{j=0}^{l} \sum_{d=0}^{r} \eta_{k, l}(-t)^{d}\left(\begin{array}{c}
r \\
d
\end{array}\right) \\
& \times\left[\frac{\gamma\left(\frac{r-d}{\theta}+l+1, \alpha(k+1) t^{\theta}\right)}{\theta(\alpha(k+1))^{\frac{r-d}{\theta}+l+1}}+\frac{\gamma\left(\frac{r-d}{\theta}+l+2, \alpha(k+1) t^{\theta}\right)}{\theta(\alpha(k+1))^{\frac{r-d}{\theta}+l+2}}\right],
\end{aligned}
$$

where $\gamma(s, t)=\int_{0}^{t} x^{s-1} e^{-x} d x$ is the lower incomplete gamma function.

\subsection{Bonferroni and Lorenz curves}

In this subsection we proposed the Bonferroni and Lorenz curves. The Bonferroni and Lorenz curves and the Bonferroni and Gini indices have applications not only in economics to study income and poverty, but also in other fields like reliability, demography, insurance and medicine. The Bonferroni and Lorenz curves are defined by

$$
\begin{aligned}
B(p) & =\frac{1}{p \mu} \int_{0}^{q} x f(x) d x \\
& =\frac{1}{p \mu} \sum_{k=0}^{\infty} \sum_{j=0}^{l} \eta_{k, l}\left[\frac{\zeta\left(\frac{1}{\theta}+l+1, \alpha(k+1) q^{\theta}\right)}{\theta(\alpha(k+1))^{\frac{1}{\theta}+l+1}}+\frac{\zeta\left(\frac{1}{\theta}+l+2, \alpha(k+1) q^{\theta}\right)}{\theta(\alpha(k+1))^{\frac{1}{\theta}+l+2}}\right]
\end{aligned}
$$

Journal home page: www.jafristat.net ; www.projecteuclid.org/as 
and

$$
\begin{aligned}
L(p) & =\frac{1}{\mu} \int_{0}^{q} x f(x) d x \\
& =\frac{1}{\mu} \sum_{k=0}^{\infty} \sum_{j=0}^{l} \eta_{k, l}\left[\frac{\zeta\left(\frac{1}{\theta}+l+1, \alpha(k+1) q^{\theta}\right)}{\theta(\alpha(k+1))^{\frac{1}{\theta}+l+1}}+\frac{\zeta\left(\frac{1}{\theta}+l+2, \alpha(k+1) q^{\theta}\right)}{\theta(\alpha(k+1))^{\frac{1}{\theta}+l+2}}\right]
\end{aligned}
$$

\subsection{Mean deviation}

In statistics, mean deviation about the mean and mean deviation about the median measure the amount of scatter in a population. For random variable $X$ with pdf $f(x)$, distribution function $F(x)$, mean $\mu=E(X)$ and $M=$ Median $(X)$, the mean deviation about the mean and mean deviation about the median are, respectively, defined by

$$
\delta_{1}(x)=\int_{0}^{\infty}|x-\mu| f(x) d x=2 \mu F(\mu)-2 \mu+2 \int_{\mu}^{\infty} x f(x) d x
$$

and

$$
\delta_{2}(x)=\int_{0}^{\infty}|x-M| f(x) d x=-\mu+2 \int_{M}^{\infty} x f(x) d x .
$$

The mean deviation of $X$ about the mean is given by

$\delta_{1}(x)=2 \mu F(\mu)-2 \mu+2 \sum_{k=0}^{\infty} \sum_{j=0}^{l} \eta_{k, l}\left[\frac{\gamma\left(\frac{1}{\theta}+l+1, \alpha(k+1) \mu^{\theta}\right)}{\theta(\alpha(k+1))^{\frac{1}{\theta}+l+1}}+\frac{\gamma\left(\frac{1}{\theta}+l+2, \alpha(k+1) \mu^{\theta}\right)}{\theta(\alpha(k+1))^{\frac{1}{\theta}+l+2}}\right]$.

The mean deviation of $X$ about the median is given by

$$
\delta_{2}(x)=-\mu+2 \sum_{k=0}^{\infty} \sum_{j=0}^{l} \eta_{k, l}\left[\frac{\gamma\left(\frac{1}{\theta}+l+1, \alpha(k+1) M^{\theta}\right)}{\theta(\alpha(k+1))^{\frac{1}{\theta}+l+1}}+\frac{\gamma\left(\frac{1}{\theta}+l+2, \alpha(k+1) M^{\theta}\right)}{\theta(\alpha(k+1))^{\frac{1}{\theta}+l+2}}\right] .
$$

\section{Estimation}

The maximum likelihood estimates (MLEs) enjoy desirable properties and can be used when constructing confidence intervals and regions and also in test statistics. Here, we determine the MLEs of the parameters of the EKPLi distribution from complete samples only. Let $x_{1} ;,,, ; x_{n}$ be a random sample of size $n$ from the EKPLi distribution given by (6). Let $\varphi=(\alpha, \theta, a, b, c)^{T}$ be $p \times 1$ vector of parameters. The total log-likelihood function for $\varphi$, say $\ell=\ell_{n}(\varphi)$, is given by

$$
\begin{aligned}
\ell= & n \log a+n \log b+n \log c+2 n \log \alpha+n \log \theta-n \log (1+\alpha) \\
& +\sum_{i=1}^{n} \log \left(1+x_{i}^{\theta}\right)+(\theta-1) \sum_{i=1}^{n} \log x_{i}-\alpha \sum_{i=1}^{n} x_{i}^{\theta}+(a-1) \sum_{i=1}^{n} \log \left(1-s_{i}\right) \\
& +(b-1) \sum_{i=1}^{n} \log \left[1-\left(1-s_{i}\right)^{a}\right]+(c-1) \sum_{i=1}^{n} \log \left\{1-\left[1-\left(1-s_{i}\right)^{a}\right]^{b}\right\}
\end{aligned}
$$


where $s_{i}=\left[1+\alpha x_{i}^{\theta} /(\alpha+1)\right] e^{-\alpha x_{i}^{\theta}}$.

The log-likelihood can be maximized either directly by using the SAS program or R-language or by solving the nonlinear likelihood equations obtained by differentiating the above equation. The associated components of the score function $U_{n}(\varphi)=\left(\frac{\partial \ell}{\partial \alpha}, \frac{\partial \ell}{\partial \theta}, \frac{\partial \ell}{\partial a}, \frac{\partial \ell}{\partial b}, \frac{\partial \ell}{\partial c}\right)^{T}=$ $\left(U_{\alpha}, U_{\theta}, U_{a}, U_{b}, U_{c}\right)^{T}$ are

$$
\begin{aligned}
& U_{\alpha}=\frac{2 n}{\alpha}-\frac{n}{1+\alpha}-\sum_{i=1}^{n} x_{i}{ }^{\theta}+(a-1) \sum_{i=1}^{n} \frac{1}{1-s_{i}}\left(\frac{x_{i}{ }^{\theta} e^{-\alpha x_{i}^{\theta}}}{(1+\alpha)^{2}}-x_{i}{ }^{\theta} s_{i}\right) \\
& +a(b-1) \sum_{i=1}^{n} \frac{\left(1-s_{i}\right)^{a-1}}{1-\left(1-s_{i}\right)^{a}}\left(\frac{x_{i}{ }^{\theta} e^{-\alpha x_{i}^{\theta}}}{(1+\alpha)^{2}}-x_{i}{ }^{\theta} s_{i}\right) \\
& -a b(c-1) \sum_{i=1}^{n} \frac{\left[1-\left(1-s_{i}\right)^{a}\right]^{b-1}\left(1-s_{i}\right)^{a-1}}{1-\left[1-\left(1-s_{i}\right)^{a}\right]^{b}}\left(\frac{x_{i}{ }^{\theta} e^{-\alpha x_{i}^{\theta}}}{(1+\alpha)^{2}}-x_{i}{ }^{\theta} s_{i}\right) \text {, } \\
& U_{\theta}=\frac{n}{\theta}-\sum_{i=1}^{n} \frac{x_{i}^{\theta} \log \left(x_{i}\right)}{\left(1+x_{i}^{\theta}\right)}-\alpha \sum_{i=1}^{n} x_{i}{ }^{\theta} \log \left(x_{i}\right)+(a-1) \sum_{i=1}^{n} \frac{s_{i} \log \left(x_{i}\right)\left(1-\alpha x_{i}{ }^{\theta}\right)}{1-s_{i}} \\
& +\sum_{i=1}^{n} \log x_{i}+a(b-1) \sum_{i=1}^{n} \frac{\left(1-s_{i}\right)^{a-1}\left[s_{i} \log \left(x_{i}\right)\left(1-\alpha x_{i}{ }^{\theta}\right)\right]}{\left[1-\left(1-s_{i}\right)^{a}\right]} \\
& +a b(c-1) \sum_{i=1}^{n} \frac{\left[1-\left(1-s_{i}\right)^{a}\right]^{b-1}\left(1-s_{i}\right)^{a}\left[s_{i} \log \left(x_{i}\right)\left(1-\alpha x_{i}{ }^{\theta}\right)\right]}{1-\left[1-\left(1-s_{i}\right)^{a}\right]^{b}}, \\
& U_{a}=\frac{n}{a}+\sum_{i=1}^{n} \log \left(1-s_{i}\right)+(b-1) \sum_{i=1}^{n} \frac{\left(1-s_{i}\right)^{a} \log \left(1-s_{i}\right)}{1-\left(1-s_{i}\right)^{a}} \\
& +b(c-1) \sum_{i=1}^{n} \frac{\left[1-\left[1-s_{i}\right]^{a}\right]^{b-1}\left(1-s_{i}\right)^{a} \log \left(1-s_{i}\right)}{1-\left[1-\left(1-s_{i}\right)^{a}\right]^{b}}, \\
& U_{b}=\frac{n}{b}+\sum_{i=1}^{n} \log \left(1-\left(1-s_{i}\right)^{a}\right)+(c-1) \sum_{i=1}^{n} \frac{\left[1-\left(1-s_{i}\right)^{a}\right]^{b} \log \left[1-\left(1-s_{i}\right)^{a}\right]}{1-\left[1-\left(1-s_{i}\right)^{a}\right]^{b}}
\end{aligned}
$$

and

$$
U_{c}=\frac{n}{c}+\sum_{i=1}^{n} \log \left(1-\left[1-\left(1-s_{i}\right)^{a}\right]^{b}\right),
$$

The maximum likelihood estimation (MLE) of $\varphi$, say $\widehat{\varphi}$, is obtained by solving the nonlinear system $U_{n}(\varphi)=0$. These equations cannot be solved analytically, and statistical software can be used to solve them numerically via iterative methods. We can use iterative techniques such as a Newton - Raphson type algorithm to obtain the estimate $\widehat{\varphi}$. For interval estimation and hypothesis tests on the model parameters, we require the information matrix.

Applying the usual large sample approximation, MLE of $\varphi$, i.e $\widehat{\varphi}$ can be treated as being approximately $N_{5}\left(\varphi, J_{n}(\varphi)^{-1}\right)$, where $J_{n}(\varphi)=E\left[I_{n}(\varphi)\right]$. Under conditions that are fulfilled 
for parameters in the interior of the parameter space but not on the boundary, the asymptotic distribution of $\sqrt{n}(\widehat{\varphi}-\varphi)$ is $N_{5}\left(0, J(\varphi)^{-1}\right)$, where $J(\varphi)=\lim _{n \rightarrow \infty} n^{-1} I_{n}(\varphi)$ is the unit information matrix. This asymptotic behavior remains valid if $J(\varphi)$ is replaced by the average sample information matrix evaluated at $\widehat{\varphi}$, say $n^{-1} I_{n}(\widehat{\varphi})$. The estimated asymptotic multivariate normal $N_{5}\left(\varphi, I_{n}(\widehat{\varphi})^{-1}\right)$ distribution of $\widehat{\varphi}$ can be used to construct approximate confidence intervals for the parameters and for the hazard rate and survival functions. An $100(1-\gamma)$ asymptotic confidence interval for each parameter $\varphi_{r}$ is given by

$$
A C I_{r}=\left(\widehat{\varphi}_{r}-z_{\frac{\gamma}{2}} \sqrt{\widehat{I_{r r}}}, \widehat{\varphi}_{r}+z_{\frac{\gamma}{2}} \sqrt{\widehat{I_{r r}}}\right)
$$

where $z_{\gamma}$ is the upper $100 \gamma$ th percentile of the standard normal distribution.

\section{Applications}

In this section, we provide two applications of the proposed EKPLi distribution to show its usefulness to fit reallife data.

\section{Example I: Cancer Patient Data.}

The first data set on the remission times (in months) of a random sample of 128 bladder cancer patients (Lee and Wang, 2003). These data have been used by Nofal et al. (2016) and Mead and Afify (2016) to fit the generalized transmuted log logistic and Kumaraswamy exponentiated Burr XII distributions, respectively.

For this data set, we shall compare the $\mathrm{t}$ of the proposed EKPLi distribution with other competitive models namely: the CGTPLi, KLi, PLi, BLi, McDonald modified Weibull (McMW) (Merovci and Elbatal, 2013) and transmuted modified Weibull (TMW) (Khan and King, 2013) models with corresponding densities (for $x>0$ ):

CGTPLi: $f(x)=\frac{\theta(1+x) \exp \left(\frac{1+\alpha+\alpha x}{1+\alpha} \theta \mathrm{e}^{-\alpha \mathrm{x}}\right)}{\alpha^{-2}(1+\alpha)\left(\mathrm{e}^{\theta}-1\right) \mathrm{e}^{\alpha \mathrm{x}}}\left\{1-\lambda \frac{\left(\mathrm{e}^{\theta}+1\right)-2 \exp \left(\frac{1+\alpha+\alpha x}{1+\alpha} \theta \mathrm{e}^{-\alpha \mathrm{x}}\right)}{\mathrm{e}^{\theta}-1}\right\}$;

BLi: $f(x)=\frac{\alpha^{2}(1+x)}{B(a, b)(1+\alpha)} \mathrm{e}^{-\alpha \mathrm{x}}\left(\frac{1+\alpha+\alpha x}{1+\alpha} \mathrm{e}^{-\alpha \mathrm{x}}\right)^{b-1}$;

McMW: $f(x)=\frac{c \alpha+c \gamma \beta x^{\beta-1}}{B(a / c, b)} \mathrm{e}^{-\alpha \mathrm{x}-\gamma \mathrm{x}^{\beta}}\left(1-\mathrm{e}^{-\alpha \mathrm{x}-\gamma \mathrm{x}^{\beta}}\right)^{a c-1}\left[1-\left(1-\mathrm{e}^{-\alpha \mathrm{x}-\gamma \mathrm{x}^{\beta}}\right)^{c}\right]^{b-1} ;$

TMW: $f(x)=\left(\alpha+\gamma \beta x^{\beta-1}\right) \mathrm{e}^{-\alpha \mathrm{x}-\gamma \mathrm{x}^{\beta}}\left(1-\lambda+2 \lambda \mathrm{e}^{-\alpha \mathrm{x}-\gamma \mathrm{x}^{\beta}}\right)$.

The parameters of the above densities are all positive real numbers except for the CGTPLi and TMW distributions for which $|\lambda| \leq 1$.

The MLEs, their corresponding standard errors (in parentheses) and the values of $-2 \widehat{\ell}, W^{*}$ and $A^{*}$ are given in Table 2 . 
I. Elbatal, Y.M.H. El Gebaly and E.A. Amin, Afrika Statistika, Vol. 11(2), 2016, pages 1075 1094. An extended power Lindley distribution and its application.

\begin{tabular}{|c|c|c|c|c|c|c|c|c|c|}
\hline \multirow{3}{*}{$\begin{array}{l}\text { Model } \\
\text { McMW } \\
(\alpha, \beta, \gamma, a, b, c)\end{array}$} & \multicolumn{6}{|c|}{ Estimates } & \multirow{3}{*}{$\frac{-2 \widehat{\ell}}{827.1}$} & \multirow{3}{*}{$\frac{W^{*}}{0.12897}$} & \multirow{3}{*}{$\begin{array}{c}A^{*} \\
0.77078\end{array}$} \\
\hline & 0.0649 & 0.6719 & 0.0008 & 0.8583 & 1.9183 & 1.3349 & & & \\
\hline & $(0.029)$ & $(0.217)$ & $\left(4.837 \cdot 10^{-3}\right)$ & $(0.322)$ & $(0.781)$ & $(0.322)$ & & & \\
\hline EKPLi & 0.6508 & 0.4822 & 1.7 & 467 & 2.2022 & 1.8297 & 821.3 & 0.04175 & 0.26919 \\
\hline$(\alpha, \theta, a, b, c)$ & $(1.316)$ & $(0.387)$ & $(5.7$ & 39) & $(8.448)$ & $(5.390)$ & & & \\
\hline TMW & 0.0612 & 1 & 7.0091 & $\cdot 10^{-7}$ & 0.8616 & & 826.9 & 0.06054 & 0.5588 \\
\hline$(\alpha, \beta, \gamma, \lambda)$ & $(0.01)$ & $\left(5.522 \cdot 10^{-4}\right.$ & $(6.158$ & $\cdot 10^{-5}$ ) & $(0.175)$ & & & & \\
\hline $\mathrm{KLi}$ & 0.0238 & 0.6201 & 8.5 & 854 & & & 834.9 & 0.19746 & 1.19401 \\
\hline$(\alpha, a, b)$ & $(0.028)$ & $(0.039)$ & (11. & 134) & & & & & \\
\hline $\mathrm{BLi}$ & 0.0232 & 0.5357 & 14.4 & 662 & & & 846.9 & 0.37053 & 2.19822 \\
\hline$(\alpha, a, b)$ & $(0.007305)$ & $(0.055)$ & $(7.4$ & 22) & & & & & \\
\hline CGTPLi & 0.0989 & 2.7639 & 0.4 & 131 & & & 821.3 & 0.06923 & 0.45551 \\
\hline$(\alpha, \theta, \lambda)$ & $(0.022)$ & $(1.204)$ & $(0.3$ & 01) & & & & & \\
\hline PLi & 0.2944 & 0.8301 & & & & & 826.7 & 0.10248 & 0.62961 \\
\hline$(\alpha, \lambda)$ & $(0.037)$ & $(0.047)$ & & & & & & & \\
\hline
\end{tabular}

Table 2. MLEs, their standard errors (in parentheses) and goodness-of-fit statistics for cancer patient data

The plots of the fitted EKPLi pdf and other fitted pdfs defined before, for the cancer data, are displayed in Figure 3. The QQ-plot of the EKPLi model and estimated cdf and sf for this model are given in Figure 4.

In Table 2, we compare the fits of the EKPLi model with the CGTPLi, TMW, McMW, PLi, KLi and BLi models. We note that the EKPLi model has the lowest values for the $-2 \hat{\ell}, W^{*}$ and $A^{*}$ statistics among the fitted models. So, the EKPLi model could be chosen as the best model. It is quite clear from the values in Table 2 that the EKPLi model provides the best fits to this data set. So, we prove empirically that this distribution can be better model than other competitive models. Further, the plots in Figure 3 reveal that the EKPLi distribution provide the best fits and it can be considered very competitive model to other distributions with positive support.

\section{Example II: Waiting Times Data.}

The second data set (Ghitany et al., 2008) consists of 100 observations on waiting time (in minutes) before the customer received service in a bank. We compare the fits of the EKPLi distribution with other competitive distributions, namely: the beta transmuted Li (BTLi) [Afify et al. (2016b)], generalized transmuted Li (GTLi) (Nofal et al., 2016), PLi, generalized Li (GLi) (Nadarajah et al., 2011), quasi Li (QLi) (Shanker and Mishra, 2013) and $\mathrm{Li}$ distributions. Table 3 lists the MLEs, their corresponding standard errors (in parentheses) and the values of $-2 \widehat{\ell}, W^{*}$ and $A^{*}$. The plots of the fitted EKPLi pdf and other fitted pdfs, for this data set, are displayed in Figure 5. Figure 6 shows the QQ-plot, estimated cdf and estimated sf of the EKPLi distribution. 

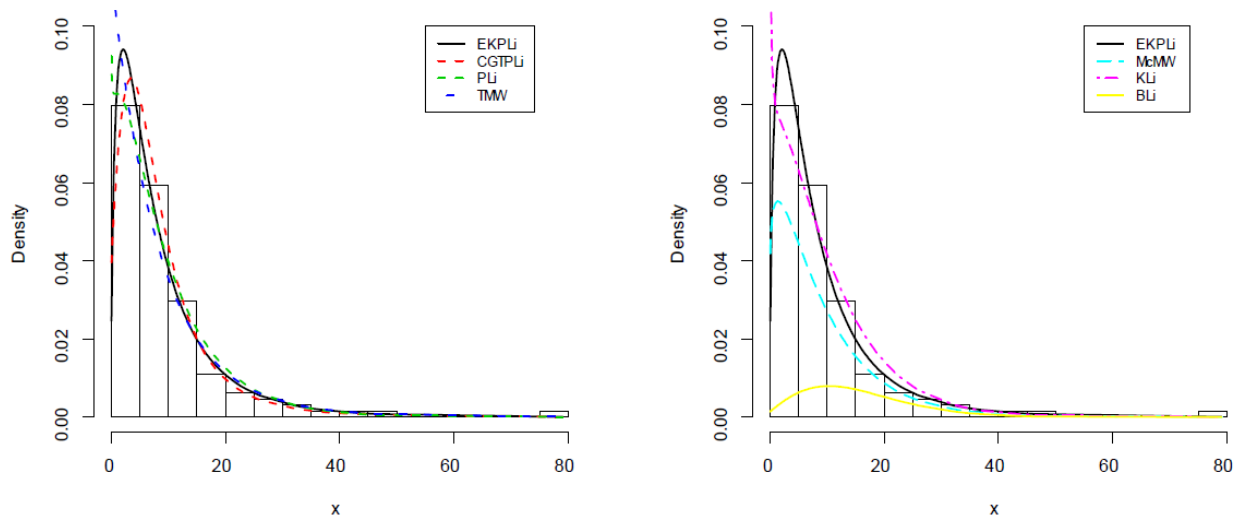

Fig. 3. The estimated EKPLi pdf and other estimated pdfs for cancer data.
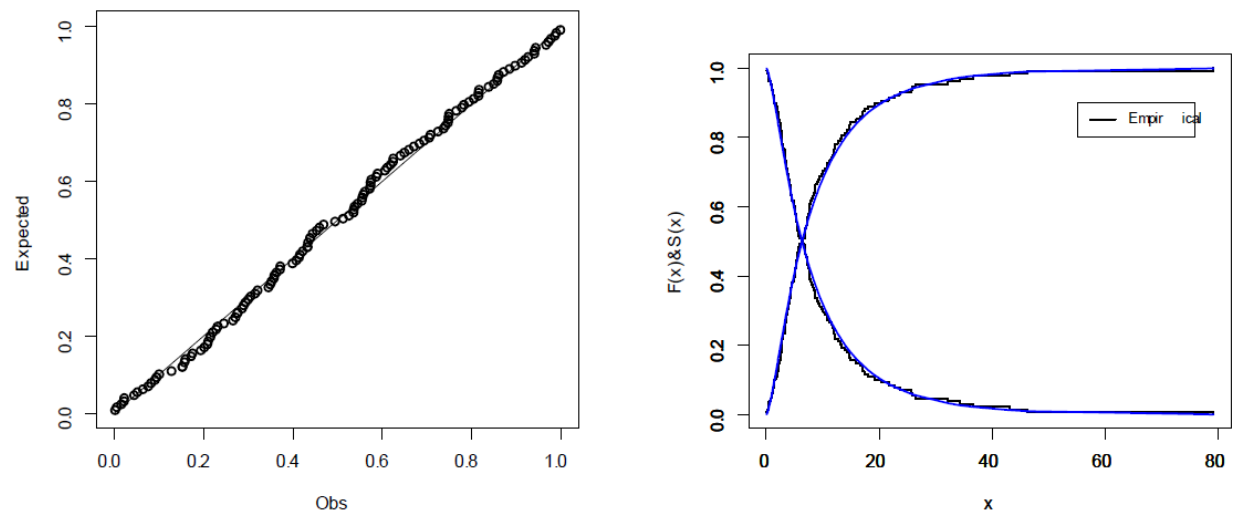

Fig. 4. QQ-plot of the EKPLi distribution (left panel) and estimated cdf

The values in Table 3 and the plots in Figures 5 and 6 reveal that the EKPLi distribution gives close fit to the waiting times data that other competitive models.

Generally, we conducted the application study to prove that the new EKPLi model is capable of modeling various data especially right skewed data comparing with some nested and non-nested models generated from well-known families.

In this sections, we used the optim function in $\mathrm{R}$. We maximized (11) using a wide range of starting values. The starting values were taken in a fine scale, for example, they were taken to correspond to all combinations of $\alpha=1,2, \ldots, 10, \theta=1,2, \ldots, 10, a=1,2, \ldots, 10$, $b=1,2, \ldots, 10$ and $c=1,2, \ldots, 10$. The call to optim converged about 98 percent of the time. When the calls to optim did converge, the maximum likelihood solution was unique.

Journal home page: www.jafristat.net; www.projecteuclid.org/as 
I. Elbatal, Y.M.H. El Gebaly and E.A. Amin, Afrika Statistika, Vol. 11(2), 2016, pages 1075 1094. An extended power Lindley distribution and its application.

\begin{tabular}{|c|c|c|c|c|c|c|c|c|}
\hline Model & & & imates & & & $-2 \widehat{\ell}$ & $W^{*}$ & $A^{*}$ \\
\hline $\begin{array}{l}\text { EKPLi } \\
(\alpha, \theta, a, b, c)\end{array}$ & $\begin{array}{l}0.1421 \\
(0.968)\end{array}$ & $\begin{array}{l}0.6859 \\
(0.759)\end{array}$ & $\begin{array}{l}0.7503 \\
(0.366)\end{array}$ & $\begin{array}{c}6.1078 \\
(32.297)\end{array}$ & $\begin{array}{l}3.7865 \\
(6.464)\end{array}$ & 634.179 & 0.01745 & 0.12575 \\
\hline $\begin{array}{l}\text { BTLi } \\
(\alpha, \lambda, a, b)\end{array}$ & $\begin{array}{l}0.1668 \\
(1.354)\end{array}$ & $\begin{array}{l}0.4374 \\
(1.086)\end{array}$ & $\begin{array}{l}1.3316 \\
(3.866)\end{array}$ & $\begin{array}{c}1.12 \\
(11.5633)\end{array}$ & & 634.801 & 0.02564 & 0.16875 \\
\hline $\begin{array}{l}\text { GTLi } \\
(\alpha, \lambda, a, b)\end{array}$ & $\begin{array}{l}0.2108 \\
(0.021)\end{array}$ & $\begin{array}{l}-0.9784 \\
(11.042)\end{array}$ & $\begin{array}{l}1.2727 \\
(0.247)\end{array}$ & $\begin{array}{l}0.0045 \\
(0.342)\end{array}$ & & 635.579 & 0.03761 & 0.24149 \\
\hline $\begin{array}{l}\mathrm{PLi} \\
(\alpha, \theta)\end{array}$ & $\begin{array}{l}0.1531 \\
(0.028)\end{array}$ & $\begin{array}{l}1.083 \\
(0.07)\end{array}$ & & & & 636.612 & 0.05114 & 0.32545 \\
\hline $\begin{array}{l}\mathrm{GLi} \\
(\alpha, \theta)\end{array}$ & $\begin{array}{l}0.2108 \\
(0.021)\end{array}$ & $\begin{array}{l}1.2771 \\
(0.196)\end{array}$ & & & & 635.579 & 0.03761 & 0.24149 \\
\hline $\begin{array}{l}\mathrm{QLi} \\
(\alpha, \theta)\end{array}$ & $\begin{array}{c}0.1025 \\
\left(5.704 \cdot 10^{-3}\right)\end{array}$ & $\begin{array}{c}83.3111 \\
(590.987)\end{array}$ & & & & 657.995 & 0.40534 & 4.47434 \\
\hline $\begin{array}{l}\mathrm{Li} \\
(\alpha)\end{array}$ & $\begin{array}{l}0.1866 \\
(0.013)\end{array}$ & & & & & 638.045 & 0.07696 & 0.57677 \\
\hline
\end{tabular}

Table 3. MLEs, their standard errors (in parentheses) and goodness-of-fit statistics for waiting times data
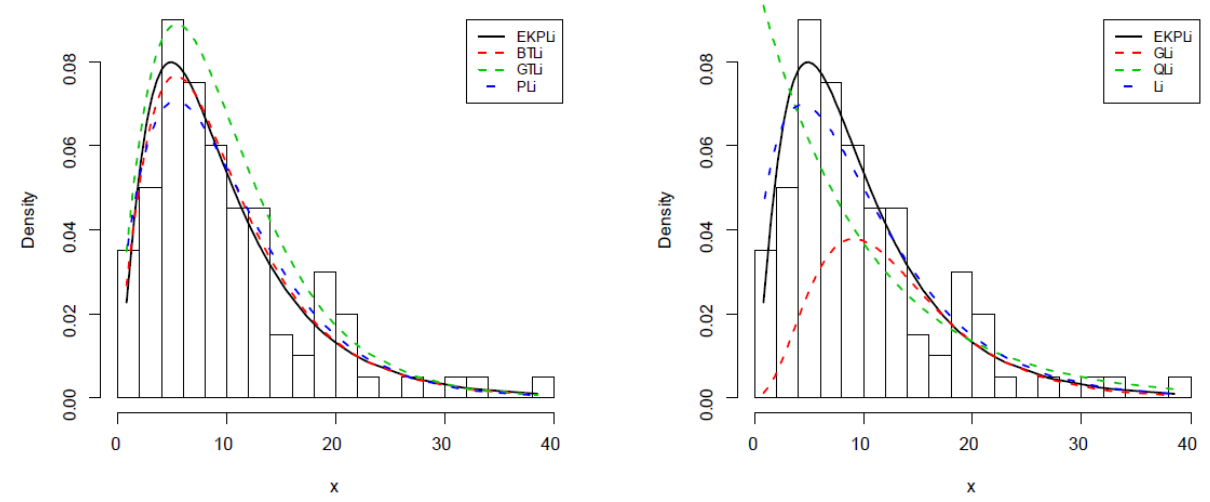

Fig. 5. The estimated EKPLi pdf and other estimated pdfs for waiting times

The unique solution was verified by using the PROC NLMIXED function in SAS. None of the unique solutions corresponded to boundaries of the parameter spaces.

\section{Conclusions}

The idea of generating new extended models from classic ones has been of great interest among researchers in the past decade. We propose a new exponentiated Kumaraswamy power Lindley (EKPLi) distribution, which extends the power Lindley (PLi) distribution by adding three extra shape parameters. Many well-known distributions emerge as special cases of the proposed model. We provide some mathematical properties of the EKPLi model including explicit expansions for the ordinary and conditional moments, residual and reversed residual life functions, Bonferroni and Lorenz curves and mean deviations. The maximum likelihood

Journal home page: www.jafristat.net; www.projecteuclid.org/as 

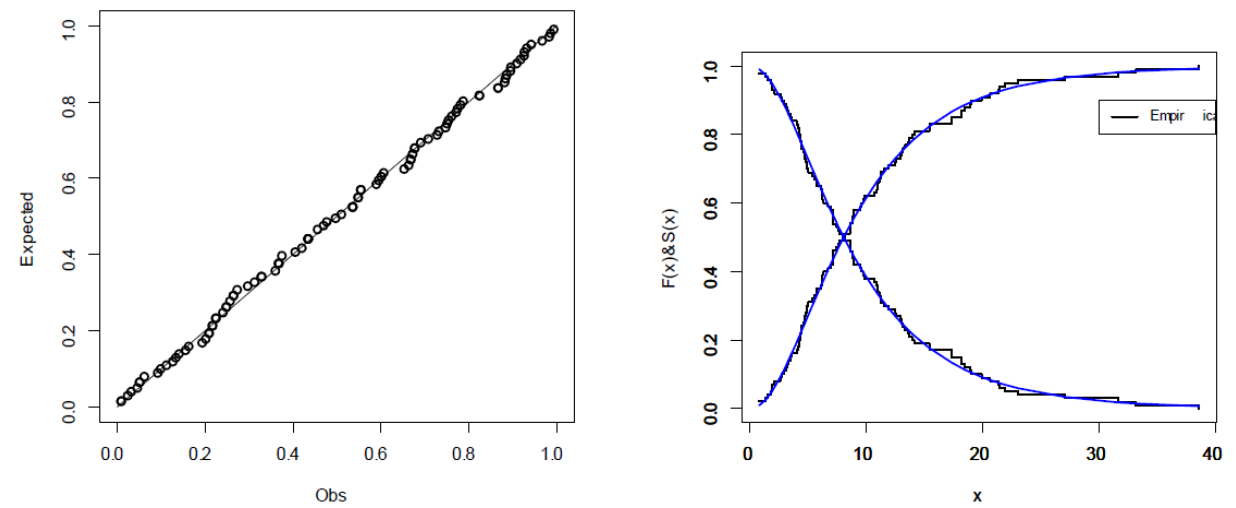

Fig. 6. QQ-plot of the EKPLi distribution (left panel) and estimated cdf

estimation of the model parameters is investigated. By means of two real data sets, we verify that the EKPLi distribution can provide better fit than other models generated from well-known families.

\section{Acknowledgments}

The authors would like to thank the Editor and the referee for careful reading and comments which greatly improved the paper.

\section{References}

Afify A.Z., Cordeiro, G.M., Butt, N.S., Ortega, E.M.M. and Suzuki, A.K. 2016. A new lifetime model with variable shapes for the hazard rate. Brazilian Journal of Probability and Statistics, forthcoming.

Afify, A.Z., Yousof, H.M. and Nadarajah, S. 2016. The beta transmuted-H family for lifetime data. Statistics and Its Interface, forthcoming.

Alizadeh, M., Yousof, H.M., Afify, A.Z., Cordeiro, G.M. and Mansoor, M., 2016. The complementary generalized transmuted Poisson-G family of distributions. Austrian Journal of Statistics. Forthcoming.

Bakouch, H.S., Al-Zahrani, B.M., Al-Shomrani, A.A., Marchi, V.A.A. and Louzada, F., 2012. An extended Lindley distribution. Journal of the Korean Statistical Society, 41, 75-85.

Cakmakyapan, S. and Kadilar, G.O., 2014. A new customer lifetime duration distribution: the Kumaraswamy Lindley Distribution. International Journal of Trade, Economics and Finance. 5, 441-444.

Cordeiro G.M. and de Castro, M., 2011. A new family of generalized distributions. Journal of Statistical Computation and Simulation. 81, 883-898.

Cordeiro, G.M., Ortega, E.M.M. and Nadarajah, S., 2010. The Kumaraswamy Weibull distribution with application to failure data. Journal of the Franklin Institute. 347, 1399-1429.

Cordeiro, G.M., Nadarajah, S. and Ortega, E.M.M., 2012. The Kumaraswamy Gumbel distribution. Statistical Methods and Applications. 21, 139-168.

Journal home page: www.jafristat.net ; www.projecteuclid.org/as 
Eugene, N., Lee, C. and Famoye, F., 2002. Beta-normal distribution and its applications. Communications in Statistics: Theory and Methods. 31, 497-512.

Ghitany, M.E, 1998. On a recent generalization of gamma distribution. Communications in Statistics-Theory and Methods. 27, 223-233.

Ghitany, M.E., Al-Qallaf, F., Al-Mutairi, D.K. and Hussain, H.A., 2011. A twoparameter weighted Lindley distribution and its applications to survival data. Math. Comput. Simulation. 81, 1190-1201.

Ghitany, M.E., Al-Mutairi, D.K., Balakrishnan, N. and Al-Enezi, L.J., 2013. Power Lindley distribution and associated inference. Computational Statistics and Data Analysis. 64, $20-33$.

Ghitany, M.E., Atieh, B., Nadarajah, S., 2008. Lindley distribution and its application. Mathematics and Computers in Simulation. 78, 493-506.

Gupta, R.D. and Kundu, D. (1999). Generalized exponential distributions. Austral. NZ J. Statist. 41, 17-188.

Gupta, P.L. and Gupta, R.C., 1983. On the moments of residual life in reliability and some characterization results. Comm. Statist. Theory Methods. 12, 449-461.

Huang, S. and Oluyede, B.O., 2014. Exponentiated Kumaraswamy-Dagum distribution with applications to income and lifetime data. Journal of Statistical Distributions and Applications. 1, 1-18.

Khan, M.S. and King, R., 2013. Transmuted modified Weibull distribution: a generalization of the modified Weibull probability distribution. European Journal of Pure and Applied Mathematics. 6, 66-88.

Kumaraswamy, P., 1980. Generalized probability density-function for double-bounded random-processes. Journal of Hydrology. 462, 79-88.

Lee, E.T. and Wang, J.W., 2003. Statistical methods for survival data Analysis. Wiley, New York.

Lemonte, A.J., Barreto-Souza, W. and Cordeiro, G.M., 2013. The exponentiated Kumaraswamy distribution and its log-transform. Brazilian Journal of Probability and Statistics. 27, 31-53.

Lindley, D.V., 1958. Fiducial distributions and Bayes theorem. Journal of the Royal Statistical Society, Series B, 20, 102-107.

Liyanage, W. and Pararai, M., 2014. A generalized power Lindley distribution with applications. Asian Journal of Mathematics and Applications. 1-23.

Mead, M.E. and Afify, A.Z., 2016. On five-parameter Burr XII distribution: properties and applications. South African Statistical Journal, forthcomig.

Merovci, F. and Elbatal, I. 2013. The McDonald modified Weibull distribution: properties and applications. arXiv preprint arXiv:1309.2961.

Merovci, F. and Elbatal, I., 2014. Transmuted Lindley-geometric and its applications. J. Statist. Appl. Probab. 3, 77-91.

Merovci, F. and Sharma, V.K., 2014. The beta Lindley distribution: properties and applications. Journal of Applied Mathematics. 51, 1-10.

Mi, J., 1995. Bathtub failure rate and upside-down bathtub mean residual life. IEEE Transactions on Reliability. 44, 388-391.

Mudholkar, G.S., Srivastava, D.K. and Freimer, M., 1995. The exponentiated Weibull family: a reanalysis of the bus-motor-failure data. Technometrics. 37, 436-445. 
Nadarajah, S., Bakouch, H.S. and Tahmasbi, R. (2011). A generalized Lindley distribution. Sankhya B. 73, 331-359.

Nadarajah, S. and Kotz, S., 2006. The exponentiated type distributions. Acta Applicandae Mathematicae. 92, 97-111.

Nedjar, S. and Zeghdoudi, H., 2016. Gamma Lindley distribution and its application. Journal of Applied Probability and Statistics. 11, 129-138.

Nofal, Z.M., Afify, A.Z., Yousof, H.M. and Cordeiro, G.M., 2016. The generalized transmuted-G family of distributions. Communications in Statistics-Theory and Methods, forthcoming.

Ozel, G., Alizadeh, M., Cakmakyapan, S., Hamedani, G.G., Ortega, E.M.M. and Cancho, V.G., 2016. The odd log-logistic Lindley Poisson model for lifetime data. Communications in Statistics Theory and Methods, forthcoming.

Pararai, M., Warahena-Liyanage, G. and Oluyede, B.O., 2015. A new class of generalized power Lindley distribution with applications to lifetime data. Theor. Math. Appl. 5, 53-96.

Park, K.S., 1985. Effect of burn-in on mean residual life. IEEE Transactions on Reliability. 34, 522-523.

Pascoa, A.R.M., Ortega, E.M.M. and Cordeiro, G.M., 2011. The Kumaraswamy generalized gamma distribution with application in survival analysis. Statistical Methodology. 8, 411433.

Rodrigues, A. and Silva, M., 2015. The exponentiated Kumaraswamy-exponential distribution. British Journal of Applied Science \& Technology. 10, 1-12.

Rodrigues, A., Silva, M. and Hamedani, G.G., 2016. The exponentiated Kumaraswamy inverseWeibull distribution with application in survival analysis. Journal of Statistical Theory and Applications. 15, 8-24.

Saulo, H., Leão, J. and Bourguignon, M., 2012. The Kumaraswamy Birnbaum-Saunders distribution. Journal of Statistical Theory and Practice. 6, 745-759.

Shanker, R and Mishra, A., 2013. A quasi Lindley distribution. African Journal of Mathematics and Computer Science Research. 6, 64-71.

Tang, L.C., Lu, Y. and Chew, E.P., 1999. Mean residual life distributions. IEEE Transactions on Reliability. 48, 68-73.

Zeghdoudi, H. and Nedjar, S., 2016. A pseudo Lindley distribution and its application. Afr. Stat. 11, 923-932. 